\title{
Triage Category Allocation and Education for Accident/Emergency Depart- ment Nurses at Referral Hospital in Kigali, Rwanda
}

\author{
Jean Claude Musengimana ${ }^{1}$, Susan Bosworth ${ }^{1}$ \\ ${ }^{1}$ King Faisal Hospital, Rwanda
}

\section{Background}

Triage is the process including the initial assessment followed by the prioritization of patients needing emergency care and assign them according to their actual need or likely benefit from immediate medical treatment. Triage is an important concept in accident and emergency (A/E) care since failure to ensure that patients are seen in a timely manner can and will result in negative outcomes, such as prolonged hospitalization, Intensive Care Unit admissions, increased resource consumption, and even death. Triage of patients at the referral hospital was complicated by existence of multiple step triage process which increased the likelihood of incorrect triage even with the use of the Australian Triage Scale (ATS). While the use of ATS triage scale may not necessarily cause delay in waiting time for consultation, inappropriate categorization could adversely influence both subsequent treatment and patient outcomes.

\section{Objectives}

To improve patient care through appropriate allocation of the ATS and verify education of A/E staff in triage protocol implementation and evaluation of current and past triage practices.

\section{Methods}

A real time motion study was conducted with 35 patients identifying waiting time from triage to doctor consultation. A retrospective chart review was conducted with evaluation of the volume of each triage category using the Australian Triage Scale (ATS), and the staff's ability to assign the appropriate ATS score was tested using a standardized written case scenario. Data collection occurred between October 2013 and July 2014. Staff education sessions reintroducing the ATS and the appropriate use based on clinical scenarios was conducted, retrospective audits of triage documentation and followed by group discussion regarding accurate triage category allocation was done. Remodeling and reorganization of the physical layout of $\mathrm{A} / \mathrm{E}$ triage nurse station was done; in order to facilitate patient flow and increase early identification of critically ill patients.

\section{Results}

There was a slight increase in categories 4 and 5 post training, but there might have been other factors such as a change in triage practice and institution of a day care room in A/E. Staff knowledge increased in accuracy of triage category allocation from $51 \%$ to $64 \%$.

\section{Conclusions}

Recommendations for the future includes quarterly chart audits and ongoing evaluation of the A/E staff, implementation and evaluation of data collection tools and their use to effectively measure triage practice, and continued orientation of new staff to the ATS system of triage.

Key words: triage category allocation and education, accident and emergency (A/E), Rwanda 